\title{
Arztgeheimnis und Datenschutz
}

\author{
Ärztinnen und Ärzte erfahren es jeden Tag: Ihre Patienten müssen sich ihnen für eine \\ erfolgreiche Behandlung vorbehaltlos anvertrauen können. Doch dieses grund- \\ legende Vertrauensverhältnis ist in jüngster Zeit unter Druck geraten. Deshalb standen \\ Arztgeheimnis und Datenschutz im Zentrum des letzten Sessionsanlasses der FMH. \\ An der Veranstaltung erläuterte Hanspeter Thür, Eidgenössischer Datenschutz- und \\ Öffentlichkeitsbeauftragter, die Thematik mit einem aufschlussreichen Referat.
}

Hanspeter Thür

Rechtsanwalt,

Eidgenössischer Datenschutzund Öffentlichkeitsbeauftragter

Korrespondenz: lic. iur. Hanspeter Thür Eidgenössischer Datenschutzund Öffentlichkeitsbeauftragter Feldeggweg 1

CH-3003 Bern

Tel. 0313224395

Fax 0313259996

www.edoeb.admin.ch
Es ist nicht überraschend, dass sich mit den grossen, notwendig gewordenen Sparanstrengungen im Gesundheitsbereich auch der Druck auf die Privatsphäre der Patienten erhöht. Es stellt sich jetzt die Frage, wie weit man gehen darf, will man weiterhin grundsätzlich am Patientengeheimnis festhalten. Ich möchte Ihnen anhand konkreter Beispiele die Problematik aus unserer Sicht erläutern.

Im letzten Sommer behandelte das Parlament das Paket betreffend die Massnahmen zur Eindämmung der Kostenentwicklung im KVG. Im Rahmen der Beratung wurde eine neue Bestimmung Art. 42 Abs. 3 bis ins KVG eingefügt. Sie hätte die Leistungserbringer verpflichtet, die Diagnose nach der Internationalen Klassifikation (ICD-10) auf der Rechnung aufzuführen. Damit wäre der bisherige Grundsatz im KVG, wonach der Patient das Recht hat, den Vertrauensarzt einzuschalten, wenn der Krankenversicherer vom Leistungserbringer eine detaillierte Diagnose verlangt, massiv aufgeweicht worden. Aus Sicht des Datenschutzes sind wir deshalb nicht unglücklich, dass das Parlament am Ende diese Vorlage - allerdings aus anderen Gründen - abgelehnt hat. Immerhin ist darauf hinzuweisen, dass der Vorschlag nicht vom Bundesrat stammte, sondern erst während der Beratung von Vertretern der Krankenversicherungen eingebracht wurde. Die Bekanntgabe der Diagnose an den Versicherer muss sicherlich noch vertieft diskutiert und überprüft werden.

Bei dieser vertieften Überprüfung ist die Frage zu beurteilen, ob ein solches Vorgehen zweckmässig und verhältnismässig ist und dem Grundsatz der Datenvermeidung entspricht. Auch das System des Tiers payant, das doch in einigen Kantonen zur Anwendung gelangt, muss hierbei einbezogen werden. Wenn die Rechnung mit der Diagnose direkt an den Versicherer geht, dann muss der Patient zwingend auf die Möglichkeit der Übermittlung an den Vertrauensarzt hingewiesen werden. Ausserdem ist die Frage zu klären, wer in einer Krankenversicherung Zugang zu solchen Rechnungen haben muss.
Ein weiterer Effekt des grossen Kostendrucks macht sich bei der Tendenz bemerkbar, dass Krankenversicherer neben der Kostenkontrolle über Managed-Care-Modelle zunehmend auch Behandlungsabläufe beeinflussen wollen. Wir haben bereits Allgemeine Gaschäftsbedingungen gesehen, die den Versicherungsnehmer verpflichten, den Behandlungsplan des Versicherers zu befolgen.

\section{Sorgen bereitet uns, dass \\ Versicherer zunehmend Einblick in die ganze Pflegedokumentation verlangen.}

Mit Bezug auf die Einführung von SwissDRG stellen sich ebenfalls einige grundsätzliche Fragen. Bekanntlich sollen unter diesem Regime grosse Datenmengen zwischen Spital und Versicherer ausgetauscht werden. Im Tarifstrukturvertrag SwissDRG (Version 0.2, genehmigt durch den Bundesrat am 18. Juni 2010) wird eine systematische Übermittlung sämtlicher tarifrelevanter Diagnose- und Behandlungscodes vorgeschrieben. Auch da stellt sich die Frage, ob die vorgesehene Regelung zweckmässig und verhältnismässig ist. Konkret stellt sich die Frage, ob die sensiblen Gesundheitsdaten tatsächlich nur jenen Personen zur Verfügung stehen werden, die sie für die Erfüllung ihrer Aufgabe wirklich benötigen

Generell gilt aus unserer Sicht: Für die Beurteilung der Wirtschaftlichkeit eines Spitals sind keine personenbezogenen Daten nötig. Im Einzelfall kann mit einer Pseudonymisierung genügend Transparenz geschaffen werden.

Für die Kodierrevisionen, die ja durch neutrale Aussenstellen durchgeführt werden, genügen aus unserer Sicht auch pseudonymisierte Daten. 


\section{Die Meinung der FMH}

eHealth: Arztgeheimnis und Datenschutz sind zu bewahren

Die elektronische Welt eröffnet auch im Gesundheitswesen viele neue Möglichkeiten - und weckt dabei immer stärker den Appetit auf Daten. Dies nicht so sehr in Bezug auf die Patientenbehandlung, sondern vor allem in Hinblick auf die Datenweitergabe. Für die $\mathrm{FMH}$ ist es ein zentrales Anliegen, diesen Datenhunger in Grenzen zu halten.

Die Möglichkeiten der Datenübermittlung und -auswertung verführen gerade die Versicherer dazu, immer mehr Daten zu fordern, ohne dafür einen klaren Verwendungszweck und Nutzen nachweisen zu können. Die FMH hat deshalb in einer ausführlichen Stellungnahme die für die Ärzteschaft grundlegenden Eckpunkte zusammengetragen:

- Arztgeheimnis und Datenschutz sind wichtig, weil der Patient sich für eine effektive Behandlung vorbehaltlos seinem Arzt anvertrauen können muss.

- Für die Kontrolle der ambulanten Leistungserbringer genügen anonymisierte Daten. Es braucht dafür keine Diagnosenangabe auf jeder Rechnung.

- Für die Kontrolle der Spitäler unter DRG nützen die Diagnosenangaben auf der Rechnung nichts. Die Kodierung kann nur durch eine externe professionelle Kodierrevision sinnvoll überprüft werden.

- Detaillierte medizinische Informationen gehören innerhalb der Versicherung ausschliesslich in die Hand des Vertrauensarztes.

Die komplette Stellungnahme der FMH finden Sie, zusammen mit konkreten Beispielen aus der Praxis, auf www.fmh.ch $\rightarrow$ Politik \& Medien $\rightarrow$ Dossiers $\rightarrow$ eHealth

Personenbezogene Daten werden nur dann benötigt, wenn eine individuelle Überprüfung einer einzelnen Leistung hinsichtlich Wirtschaftlichkeit und Zweckmässigkeit durchgeführt werden soll. Die stan- dardmässige Überprüfung der Rechnungen (Ausreisser-Kontrolle) kann grundsätzlich auch mit pseudonymisierten Daten durchgeführt werden. Im Bedarfsfall kann dann eine Zuordnung erfolgen. Sorgen bereitet uns auch, dass Versicherer zunehmend Einblick in die ganze Pflegedokumentation verlangen.

Grundsätzlich wird für SwissDRG eine Lösung gefunden werden müssen, die der Rechtsprechung des Bundesverwaltungsgerichts entspricht. Das Bun-

\section{Heikle Informationen sollten nur an den Vertrauensarzt der Versiche- rung übermittelt werden dürfen.}

desverwaltungsgericht hat in seinem Entscheid vom 29. Mai 2009 (C-6570/2007) einerseits festgehalten, dass eine systematische Übermittlung von Diagnoseund Eingriffcodes in einem Tarifvertrag für die Überprüfung der Wirtschaftlichkeit der Leistung geregelt werden kann. Andererseits hat es ganz klar betont, dass auch Regelungen getroffen werden müssen, damit das Verhältnismässigkeitsprinzip und das Prinzip des geringstmöglichen Eingriffs respektiert werden. Hier sind wir ganz klar der Meinung, dass heikle Informationen, eben gesellschaftlich stigmatisierende Diagnosen, nur an den Vertrauensarzt der Versicherung übermittelt werden dürfen. Dies muss im Sinn von «privacy by design» automatisch erfolgen und nicht nur in den Fällen, wo es der Patient ausdrücklich verlangt.

\section{Fazit}

Wir beobachten in zahlreichen Bereichen aus Kostengründen einen massiven Druck auf das Patientengeheimnis. Die Versicherer nehmen zunehmend auch Einfluss auf den Behandlungsablauf. Aus Sicht des Persönlichkeitsschutzes ist bei dieser Gelegenheit darauf hinzuweisen, dass in diesem sehr heiklen Bereich Regelungen nicht nur durch die Brille der Kostenersparnis beurteilt werden dürfen. Es darf nicht so weit kommen, dass am Ende das Patientengeheimnis nur noch eine leere Hülle darstellt. 